\title{
Three-dimensional finite element analysis of the human middle ear and an application for clinics for tympanoplasty
}

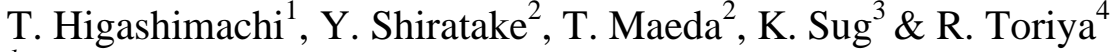 \\ ${ }^{1}$ Department of Mechanical Engineering, Sojo University, Japan \\ ${ }^{2}$ Sojo University, Japan \\ ${ }^{3}$ Suga Dental Clinic, Japan \\ ${ }^{4}$ Toriya ENT Clinic, Japan
}

\begin{abstract}
The geometric model around the middle ear which includes the tympanic membrane, tympanic cavity, auditory ossicles, several ligaments, and tensor was constructed using SolidWorks. The auditory ossicles consist of malleus, incus and stapes. The computerized tomography (CT) scan data around the middle ear was converted into DICOM (Digital Imaging and Communication in Medicine) data, then into STL data. This STL data was imported to SolidWorks in order to generate the geometric model. The sound pressure through the tympanic membrane was applied to this model in a three-dimensional finite element analysis using COSMOSWorks.

Then, the mechanical influence of the sound pressure upon the middle ear was analyzed. The deformation of the middle ear and the displacement of the stapes under the sound pressure of $120 \mathrm{~dB}$ were clarified. The displacement of the bottom of the stapes in the direction of the axis is about 3.1 nanometers which becomes a standard value of the hearing ability evaluation. In the internal ear, the stapes vibration is transmitted to the labyrinthine fluid in the cochlea where electrical signals are generated. Finally, it is recognized in the brain as sound. For example, in the case that the medical device is substituted for the deficient auditory ossicles, it is possible to estimate hearing ability by comparing to what degree the displacement of stapes changes. This kind of approach makes it possible to propose a new medical treatment for the recovery of conductive hearing loss.
\end{abstract}

Keywords: geometric model, SolidWorks, FEM, human middle ear, tympanic membrane, auditory ossicles, tympanoplasty operation, sound pressure. 


\section{Introduction}

In order to inspect our hypothesis that stress concentration is closely related to the cause of dental caries, finite element structural analysis of teeth and periodontium was carried out. Fig. 1 shows the finite element model for teeth analysis. This model consists of the primary premolar tooth, the secondary premolar tooth, the periodontal membrane, and the alveolar bones. Fig. 2 shows Mises stress contour lines of the vertical section of the model. It became clear that the stress concentration occurred just under the contact point, which was similar to the actual growth point of the dental caries. Furthermore, it was clarified that stress concentration was closely related to the eminence of the alveolar bone, attrition, and wedge-shaped defects [1].

Therefore it can be analogized that loads through the temporomandibular joint (TMJ) have some effects on the external, middle and inner ear. For instance, sometimes, TMJ disorder causes severe pain and trouble opening the mouth. In addition, it causes vertigo, tinnitus and hearing loss. However, the causes of ringing in the human ear, hearing loss and other ear diseases have not yet been clarified.

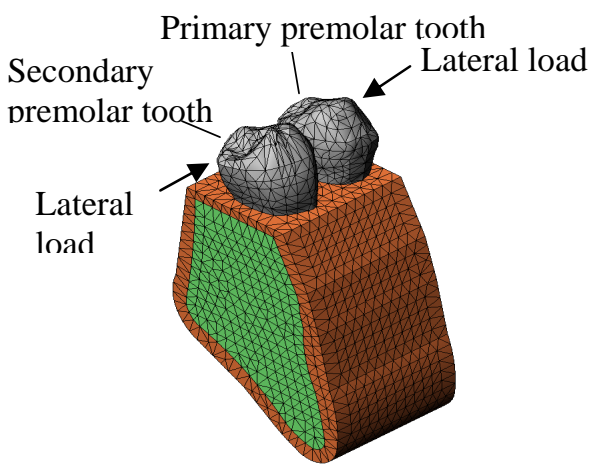

Figure 1: $\quad$ Finite element model for teeth analysis.

A structural analysis model including the TMJ, the external auditory canal, the tympanic membrane, the auditory ossicles, and the cochlea was constructed. The auditory ossicles consist of malleus, incus and stapes. The articular disc is located between the head of the mandible and the articular fossa of the temporal bone. Loads from the TMJ are transmitted to the external canal through the contact surface of the articular disc. Fig. 3 shows the finite element model for the ear analysis [2]. Fig. 4 shows that remarkable stress concentrations occur in regions $\mathrm{A}$ and $\mathrm{B}$ of the stapes crura of auditory ossicles. These positions are places destroyed by chronic otitis media and middle ear cholesteatoma. The defect becomes a cause of conductive hearing loss.

On the other hand, various sound pressures are transmitted to auditory ossicles through the tympanic membrane. The research of Koike and Wada [3] 


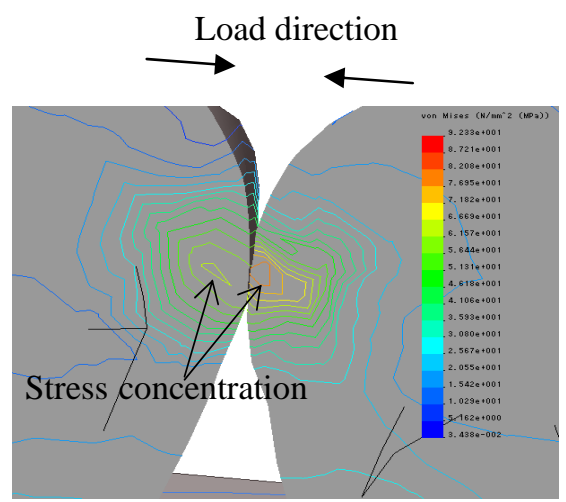

Figure 2: $\quad$ Mises stress contour of vertical section.

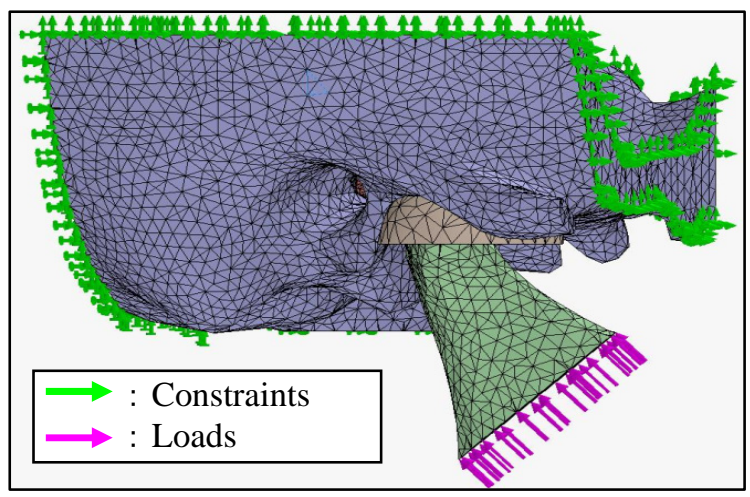

Figure 3: $\quad$ Finite element mesh and boundary conditions.

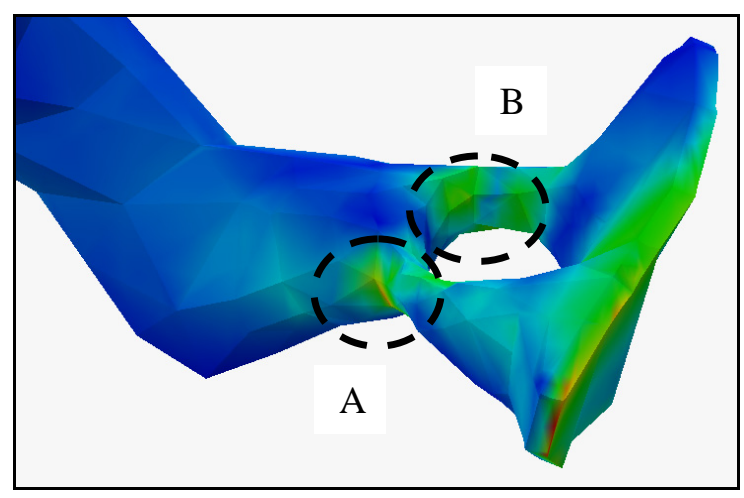

Figure 4: $\quad$ Stress concentration around stapes crura. 
concerns the vibration analysis of auditory ossicles. However, there are few structural analysis examples of the auditory ossicles from the viewpoint of mechanics. In this study, the geometric model of the middle ear and the neighbouring organ is constructed by using SolidWorks based on the CT scan data. Then, the deformation, displacement and stress of the auditory ossicles are clarified using COSMOSWorks. We assumed that the displacement of the stapes in the axial direction is closely related with hearing ability. The investigation to determine whether this assumption is good or not is carried out. The four models which can be utilized for the operation of hearing improvement are constructed, and those effects are verified.

\section{Middle ear structure and its function}

\subsection{Middle ear structure}

The middle ear is composed of the tympanic membrane, tympanic cavity, auditory ossicles and others which are shown in fig. 5. Auditory ossicles are behind the tympanic membrane in a small space (tympanic cavity), and they are composed of malleus, incus and stapes. The stapes basal plane connects with the inner ear through the oval window.

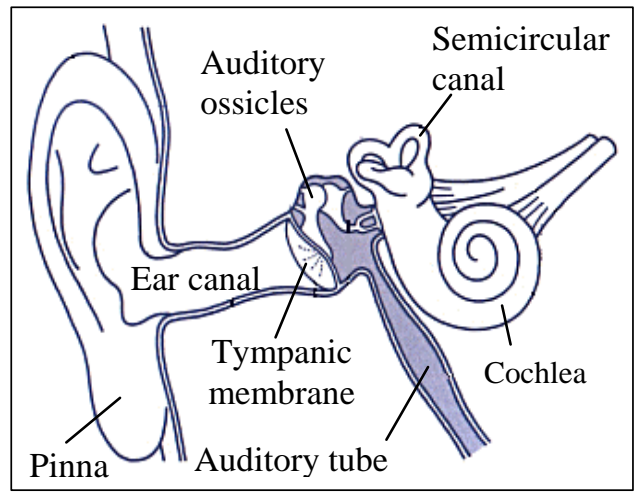

Figure 5: $\quad$ Middle ear structure.

\subsection{Function of tympanic cavity}

The tympanic cavity is the space filled with air. The inner wall of the tympanic cavity is covered with the mucous membrane.

The air pressure of the tympanic cavity is controlled at the appropriate value by ventilation in order to keep the important function that is "the sense of hearing”. Furthermore, the tympanic cavity has the washing function that absorbs and removes bacteria waste by the secretion and reabsorption of the mucus. 


\subsection{Function of auditory ossicles}

Each part of the auditory ossicles is connected with the joints. They are suspended by ligaments and muscles in the tympanic cavity. The vibration amount of the tympanic membrane is amplified about 17 times by the area ratio of stapes basal plane and the tympanic membrane. In addition, the vibration amount of the tympanic membrane is amplified about 1.3 times by the lever motion of the auditory ossicles.

\section{Finite element analysis}

\subsection{Geometric modelling}

The geometric model for the finite element analysis is shown in fig. 2. This model is composed of 14 kinds of parts. The CT scanning data of the human head except the stapedial annular ligament were converted to DICOM data, and in addition, converted into STL data which were imported into SolidWorks. The annular ligament was made in order to adjust to the geometric model of the stapes. Material data of each part are shown in table 1 . The parts name number in the table corresponds to the number in fig. 6 .

Table 1: $\quad$ Material data.

\begin{tabular}{|c|c|c|}
\hline Parts name & $\begin{array}{l}\text { Young's } \\
\text { modulus } \\
{[\mathrm{MPa}]}\end{array}$ & $\begin{array}{l}\text { Poisson's } \\
\text { ratio }\end{array}$ \\
\hline (1)Tympanic cavity wall & \multirow{4}{*}{13,436} & \multirow{14}{*}{0.3} \\
\hline (2)Malleus & & \\
\hline (3)Incus & & \\
\hline (4) Stapes & & \\
\hline (5)Lateral malleus ligament & \multirow{3}{*}{21} & \\
\hline (6)Upper malleus ligament & & \\
\hline (7)Anterior malleus ligament & & \\
\hline 8Incudomallear joint & \multirow{2}{*}{6} & \\
\hline (9)Incudostapedial joint & & \\
\hline (10)Upper incudal ligament & \multirow{3}{*}{0.65} & \\
\hline (11)Posterior incudal ligament & & \\
\hline (12)Stapedial annular ligament & & \\
\hline (13)Tympanic membrane & 64 & \\
\hline (14) Stapedial muscle & 0.52 & \\
\hline
\end{tabular}




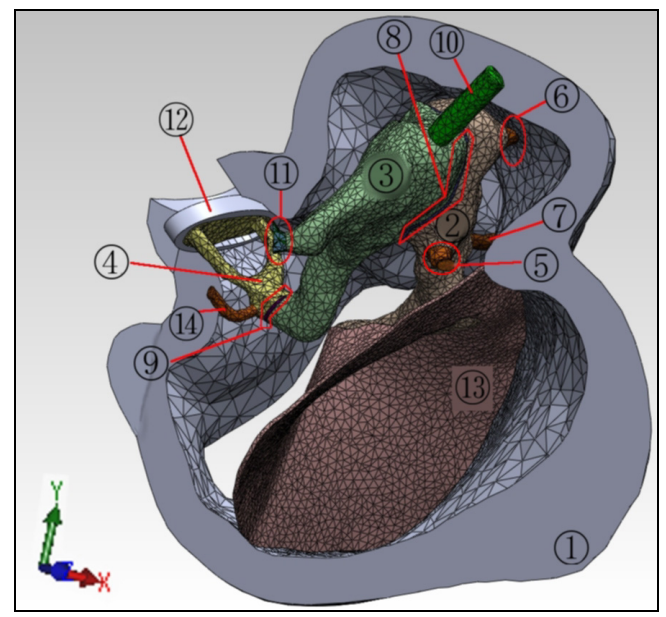

Figure 6: Geometric model of the middle ear.

\subsection{Boundary condition}

A total of 3 cross sections of tympanic cavity were perfectly fixed in constraint conditions. The sound pressure of $120 \mathrm{~dB}$ was converted into pressure using the following equation as load conditions.

$$
L_{p}=20 \log _{10} \frac{P}{P_{0}}
$$

where,

$L_{p}=120(\mathrm{~dB})$ : the limit sound pressure which human auditory sense is able to hear safely.

$P_{0}=20 \times 10^{-6}(\mathrm{~Pa})$ : standard value (the lowest value of the sound intensity which is audible for humans).

As a result, the pressure of $P=20(\mathrm{~Pa})$ was given at the contact surface of the tympanic membrane and malleus.

\subsection{Finite element analysis results}

The deformation of auditory ossicles is shown in fig. 7. Fig. 8 shows the displacements of stapes bottom in the Z-direction which is perpendicular to the basal plane.

\section{An example of clinical application}

\subsection{A predictive method of hearing ability improvement}

Fig. 7 shows that the auditory ossicles turn about the axis connecting the upper malleus ligament and the posterior incudal ligament. By this rotary motion, the 
vibration of tympanic membrane is efficiently converted into the Z-direction movement of stapes basal plane. The stapes vibration is transmitted to the labyrinth fluid of the internal ear and converted to electrical signals, then recognized as sound in the brain. In this study, we consider that there is a certain relationship between hearing ability and the Z-direction displacement of the stapes.

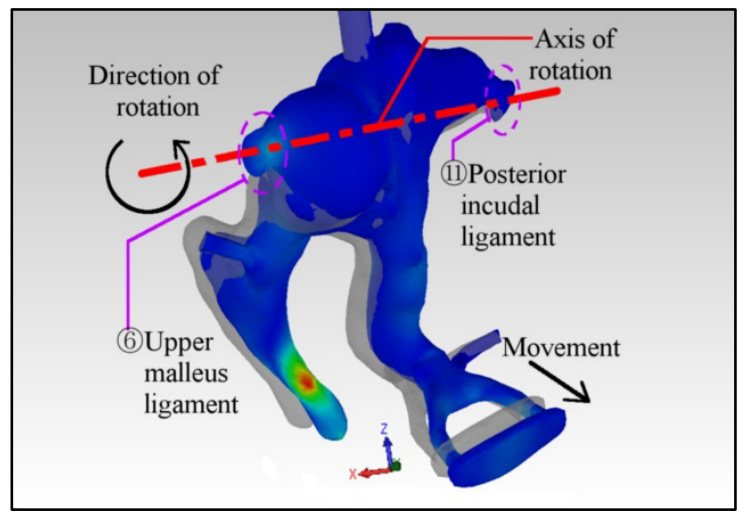

Figure 7: Deformation of auditory ossicles.

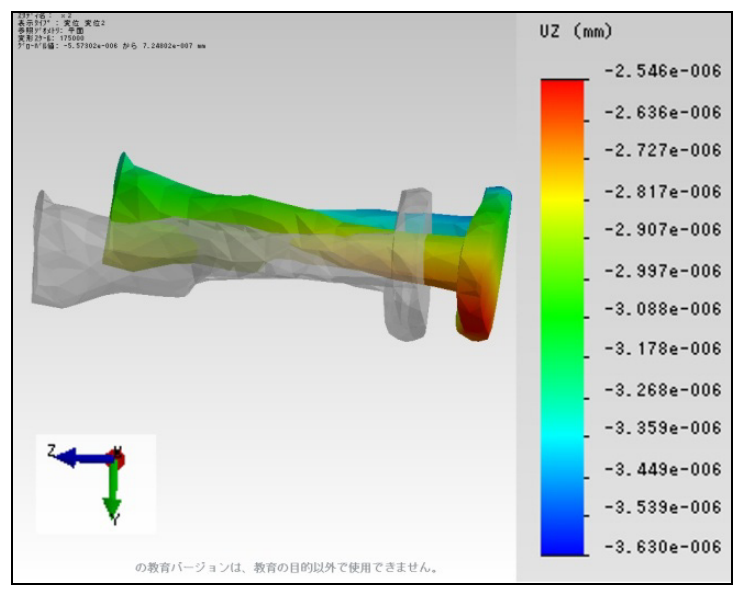

Figure 8: $\quad$ Z-direction displacement of stapes.

Fig. 8 shows that the average displacement of the stapes base for the sound pressure of $120 \mathrm{~dB}$ is $3.09 \times 10^{-6}$ [mm] which becomes a standard value in this study. If the change of this displacement is compared, the hearing restoration effect can be estimated, in case the medical device is substituted for the deficient auditory ossicles, or in the case of the stiffening of the ligament. 


\subsection{Operation model for hearing ability improvement}

\subsubsection{Tympanoplasty type with columella}

Examples of the operation of hearing ability improvement applied to the case in which auditory ossicles were damaged for chronic otitis media, etc. are shown below. One is the operation model called "Auditory ossicles formation III-C type", and the other is the operation model called "Auditory ossicles formation IV-C type". The III type means the condition in which the stapes is kept in is almost normal. The IV type is the condition in which only the basal plane of the stapes is left.

The cylindrical part called the columella is installed between the tympanic membrane and the stapes, in case the incus has been damaged. The schematic view and constructed geometric model of III-C and IV-C type are shown in fig. 9 and fig. 11 [4]. The material data of columella is the same as ceramics. Young's modulus is $E=220,590$ ( $\mathrm{MPa}$ ) and Poisson's ratio is $v=0.22$. On the other hand, the material data of junction is $E=6(\mathrm{MPa})$ and $v=0.3$. The analytical results using COSMOSWorks are shown in fig. 10 and fig. 12. Table 2 shows the average value of Z-direction displacements in each model.

The displacement of III-C type model is $1.34 \times 10^{-6}(\mathrm{~mm})$ which is about $43.4 \%$ of the normal model. The displacement of IV-C type model is $1.19 \times 10^{-6}$ (mm) which is about $38.5 \%$ of the normal model. These results almost correspond with the hearing ability recovery rate of the operation. Furthermore, the displacement of IV-C model is about $11 \%$ less than the III-C model. Because columella is connected to the normal stapes in the case of the III type model, it follows that the vibration of the tympanic membrane is easier to reach the stapes than the IV type model with damaged stapes except its bottom plate.

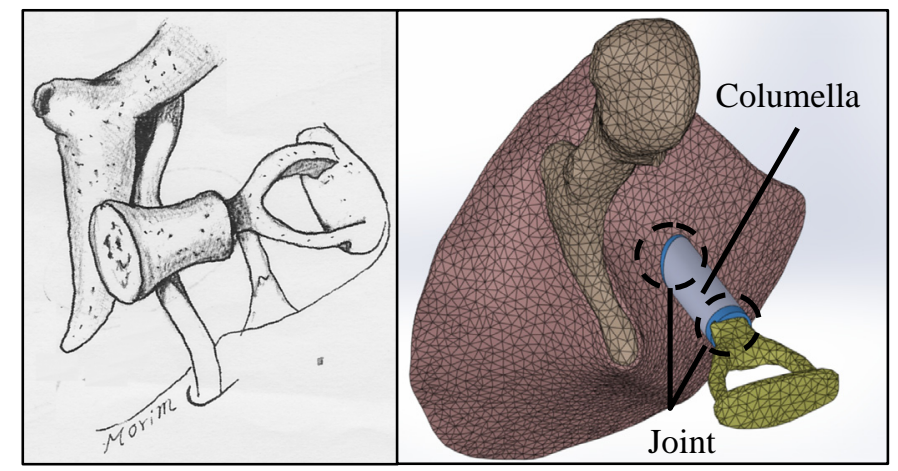

Figure 9: $\quad$ Schematic view and geometric model of III - C type. 


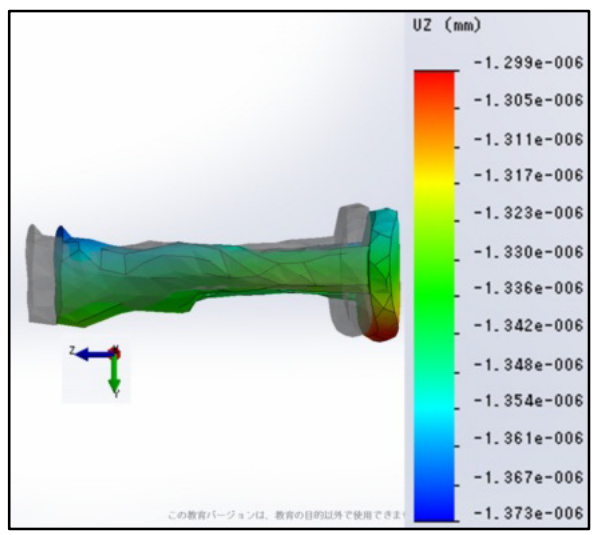

Figure 10: Z-direction displacement of stapes in III-C type.

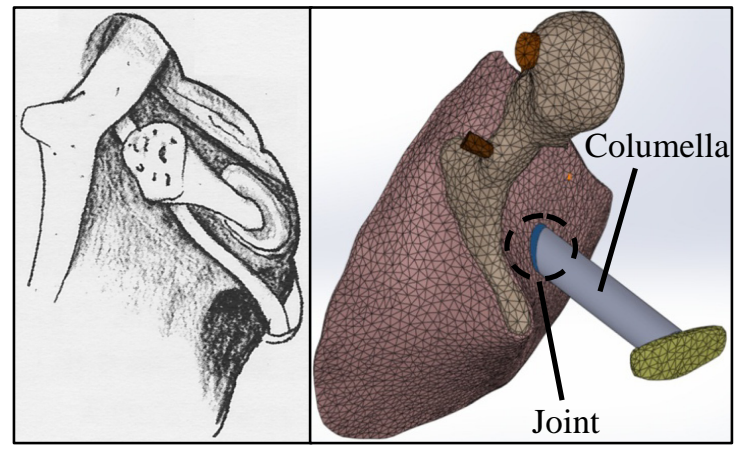

Figure 11: $\quad$ Schematic view and geometric model of IV-C type.

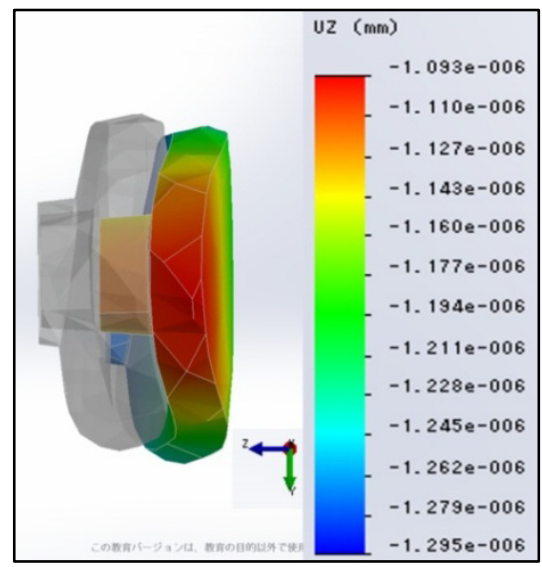

Figure 12: Z-direction displacement of stapes in IV-C type. 
Table 2: $\quad$ Average displacement of stapes.

\begin{tabular}{|c|c|c|c|}
\hline Type & Healthy & III-C & IV-C \\
\hline displacement[nm] & 3.09 & 1.34 & 1.19 \\
\hline ratio to healthy type & 1 & 0.434 & 0.385 \\
\hline
\end{tabular}

\subsubsection{Tympanoplasty type with interposition}

Other examples of tympanoplasty operation are shown below. One is the operation model called "Auditory ossicles formation III-i type" and the other is the operation model called "Auditory ossicles formation IV-i type". Cylindrical columella is installed between the malleus and stapes instead of the damaged incus. The schematic view and constructed geometric model of III-i type and IVi type are shown in fig. 13 and fig. 15 [4]. Analytical results using COSMOS Works are shown in fig. 14 and fig. 16. Table 3 shows the average value of $\mathrm{Z}$ direction displacement in each model.

The displacement of III-i type model is $3.87 \times 10^{-6}(\mathrm{~mm})$ which is about $125 \%$ of the normal model. The average value of $\mathrm{Z}$-direction displacements in IV-i type model is about $2.56 \times 10^{-6}(\mathrm{~mm})$ which is about $82.8 \%$ of the normal model. These results almost correspond with the hearing ability recovery rate of the operation. Furthermore, the displacement of IV-i model is about 33.6\% less than III-i model. This difference arises due to similar reasoning as the case of columella type model III-C, IV-C) in the preceding clause 4.2.1.

On the other hand, because columella is connected to the hard malleus in the case of the interposition model (III-i, IV-i), it follows that the vibration of the tympanic membrane is easier to reach the stapes than the columella model (III-C, IV-C). These results are appropriate in the clinical viewpoint.

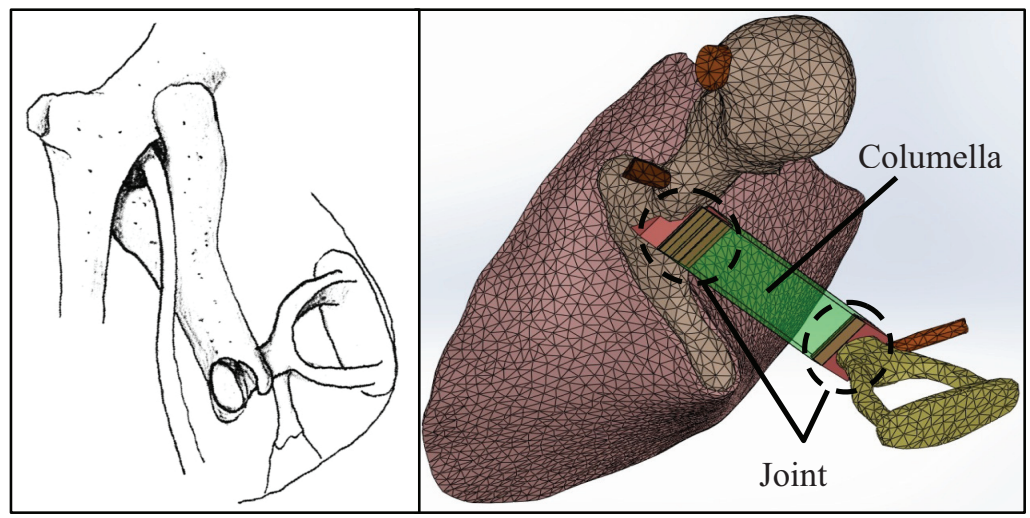

Figure 13: Schematic view and geometric model of III-i type. 


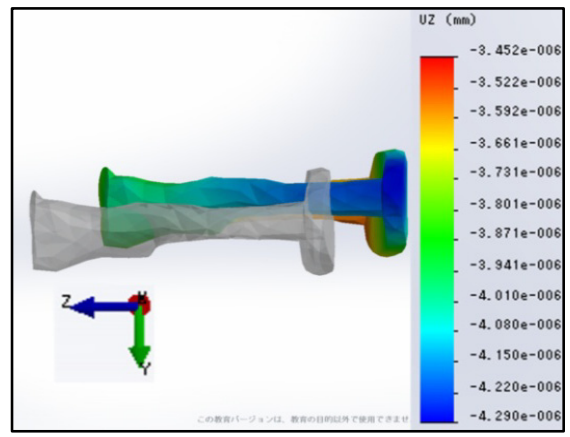

Figure 14: $\quad$ Z-direction displacement of stapes in III- i type.

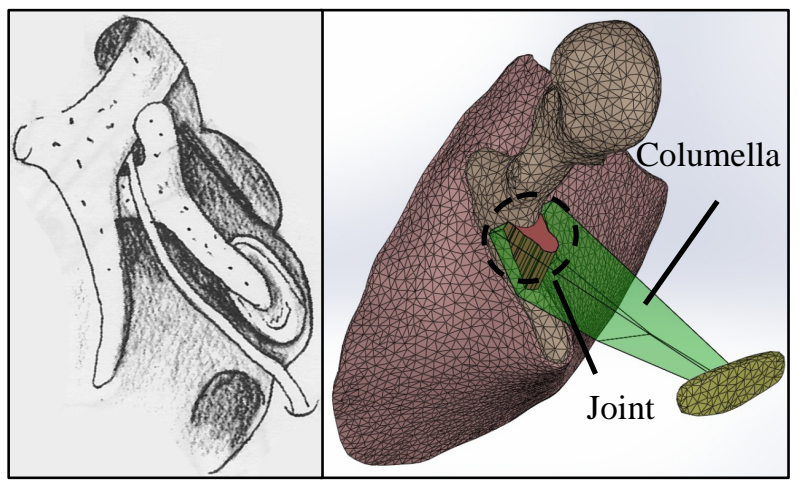

Figure 15: Schematic view and geometric model of IV- i type.

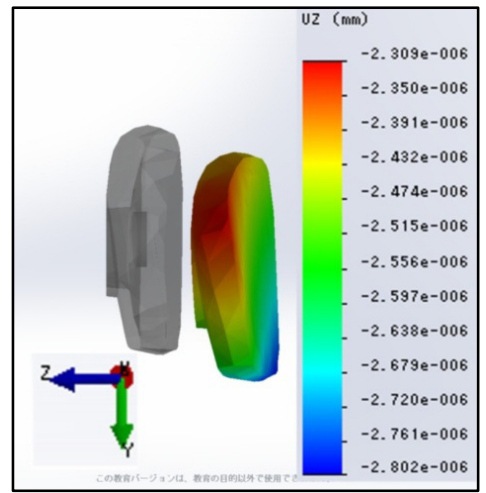

Figure 16: Z-direction displacement of stapes in IV-i type. 
Table 3: $\quad$ Average displacement of stapes.

\begin{tabular}{|c|c|c|c|}
\hline Type & Healthy & III-i & IV-i \\
\hline displacement[nm] & 3.09 & 3.87 & 2.56 \\
\hline ratio to healthy type & 1 & 1.25 & 0.828 \\
\hline
\end{tabular}

\section{Conclusions}

This study using the finite element analysis clarified the deformation and displacement of the auditory ossicles under sound pressure through the surface of the tympanic membrane. The four models which can be utilized for the operation of hearing improvement are constructed, and those effects were verified. As a result, the following knowledge was obtained.

(1) The detailed geometric model around the middle ear which includes tympanic membrane, tympanic cavity, auditory ossicles, several ligaments, and tensor was constructed using SolidWorks.

(2) It was possible to reproduce the motion of auditory ossicles in the sound conduction by the structural analysis model using COSMOSWorks. It was ascertained that the auditory ossicles turned about the axis connecting the upper malleus ligament and the posterior incudal ligament. According to this motion, the stapes basal plane moves back and forth.

(3) The axial displacement of the stapes bottom equals about 3.09 nanometers which becomes a standard value under sound pressure of $120 \mathrm{~dB}$. If the change of this displacement magnitude is compared, the hearing restoration effect can be estimated. That is, it is possible to compare to what degree the displacement of stapes changes in case the medical device is substituted for the deficient auditory ossicles.

(4) The effect of tympanoplasty with interposition type (III-i and IV-i) is better than the columella type (III-C and IV-C). On the other hand, the effect of III type model (III-i and III-C) is better than IV type model (IV-i and IV-C). These results are appropriate in the clinical viewpoint.

(5) This kind of approach makes it possible to propose a new medical treatment for the recovery of conductive hearing loss.

\section{References}

[1] Higashimachi, T., Suga K. and Sasahara H., Structural mechanical analysis and evaluation for extension of teeth lifetime. Journal of the Japan Society for Precision Engineering, 75(3) pp.418- 423, 2009.

[2] Higashimachi, T., Toriya, R. and Suga, K., Finite Element Analysis of External, Middle and Inner Ear under Loads Through Temporomandibular Joint. Key Engineering Materials, 516, pp.457-462, 2012.

[3] Koike, T. and Wada, H., Modeling of the human middle ear using the finiteelement method. Journal of Acoustical Society of America, 111(3), pp.13061317, 2002.

[4] Morimitsu, T., Illustrated Ear Surgery, Medical Illust Co.: Tokyo, Japan, 1979. 